\title{
A risk analysis of step-down equity-linked securities based on regime-switching copula
}

\author{
Manh Duc Nguyen ${ }^{a}$, Bangwon Ko $^{a}$, Hyuk-Sung Kwon ${ }^{1, a}$ \\ ${ }^{a}$ Department of Statistics and Actuarial Science, Soongsil University, Korea
}

\begin{abstract}
The globalization of financial markets has broadened investment opportunities. International investors' investment portfolios consist of financial instruments from various countries; consequently, the risks associated with economic dependence among countries should be carefully considered. Step-down equity-linked securities (ELS) are a structured financial product that have recently become popular among Korean investors. Payoffs are based on two or three stock indices from different regions; therefore, dependence between the indices should be reflected in the risk analysis. In this study, we consider a regime-switching copula model to describe the joint behavior of two stock indices- the Eurostoxx50 and the Hang Seng China Enterprises Index (HSCEI). These indices are commonly used as underlying assets of step-down ELS. Using historical data, we analyze the risk associated with step-down ELS through the probabilities of early redemption. A regime-switching copula model can accommodate complicated dependence. Thus, it should be considered in the risk analysis of step-down ELS.

Keywords: dependence model, regime-switching copula, regime-switching log-normal model, stepdown equity-linked security (ELS), structured product
\end{abstract}

\section{Introduction}

The globalization of financial markets has increased investment opportunities such that investors in one country can take advantage of favorable economic conditions in other countries. However, risks resulting from economic interdependence and increased connections between economies in different countries is an important concern as international investment becomes more popular. A negative economic shock in one country can quickly spread to other countries. For example, the 2008 global financial crisis, which arose from sub-prime mortgages in the US, affected almost all countries. Therefore, in financial risk analysis, consideration of the dependence among countries is indispensable.

Recent innovation in the financial securities market that has resulted from developments in financial engineering has provided a variety of international investment opportunities. A popular financial product in Korea is step-down equity-linked securities (ELS), which is a type of auto-callable structured product. Guillaume (2015) discusses the value, payoff structure, and relevant risk management issues of auto-callable structured products. The author presented a formula that can estimate the probability of early redemption and expected return of auto-callable structured products. Deng et al. (2011) derived a partial differential equation to model auto-callable structured products. The authors illustrated the pricing of a popular auto-callable product and estimated the probability of early redemption at each auto-call date. Lee et al. (2019) derived a closed-form formula that can be used for pricing step-down ELS for which the underlying asset is a single index value.

\footnotetext{
${ }^{1}$ Corresponding author: Department of Statistics and Actuarial Science, Soongsil University 369 Sangdo-ro, Dongjak-gu, Seoul 06978, Korea. E-mail: hskwon@ssu.ac.kr

Published 31 January 2020 / journal homepage: http://csam.or.kr (c) 2020 The Korean Statistical Society, and Korean International Statistical Society. All rights reserved.
} 
Kang (2016) analyzed investment benefits of step-down ELS from the perspective of portfolio investment involving structured products. The author used a simulation approach based on time series model to derive the distribution of return of auto-callable ELS. Kim and Yeo (2011) quantified the possibility of early redemption and relevant risk measure in step-down ELS having Korea Composite Stock Price Index 200 (KOSPI200) and Hang Seng China Enterprises Index (HSCEI). Lim and Choi (2015) discussed a hedging strategy for shortfall risk associated with knock-in feature of step-down ELS. Further, Lee et al. (2016) analyzed risk inherent in step-down ELS having two underlying indices and developed another hedging strategy based on conditional value at risk (CVaR).

The step-down ELS's payoff also depends on the values of the stock indices for two different geographic regions, which are reasonably considered to be dependent. Therefore, modeling the correlated variations in values of underlying stock indices in a risk analysis of step-down ELS should reflect two aspects: The first is the degree of dependence between the two stock indices and the other is the possible change in the degree of dependence over time.

The copula model is used to construct the joint distribution of two or more dependent random variables. The various types of copula are characterized by the dependence structure they can accommodate. Therefore, it is anticipated that copulas would be useful for modeling the structure and the degree of dependence between two stock indices for step-down ELS. Issues relating to dependent risks and copula have been addressed at length in several previous studies such as Denuit et al. (2005) and Nelson (2006). Genest and Favre (2007) also illustrate the implementation of the copula model for two dependent random variables using hydrological data. Jondeau and Rockinger (2006) observed the dependence among four major stock market indices using copula models. The authors found a strong and consistent dependence between European stock market indices. Other applications of the copula model to investigate the relationship between a stock index and other economic variables can be found in Sukcharoen et al. (2014), Delatte and Lopez (2013), and Nguyen et al. (2016).

Additionally, when different models need to be considered for different environments or conditions, a regime-switching model allows for various modes, which are called regimes, of dependence structure corresponding to the changing environment. This phenomenon can be modeled by a regimeswitching model if the degree of dependence and/or dependence structure of underlying indices of step-down ELS is thought to change with global economic conditions. The model is discussed comprehensively in Hardy (2003). Applications of the regime-switching model for stock returns can be found in Cai (1994), Hardy (2001), and Schaller and Norden (2010).

Incorporating copula into the framework of a regime-switching model, also known as a regimeswitching copula, allows us to model possible changes in the dependence structure of multiple assets. For example, the variations of several stock indices, whose dependence largely stem from global economic conditions, can be modeled more efficiently as this model allows for various modes of dependence structure by period. Therefore, the risk of step-down ELS can be analyzed by modeling the two underlying stock indices using a regime-switching copula. Chollete et al. (2008) applied regimeswitching copula to model asymmetric dependence among international financial market returns. The authors invented a method of constructing a copula that flexibly reflects the dependence structure of multiple assets.

Zhu et al. (2016) recently utilized a regime-switching copula to model the time-varying dependence structure of assets in a portfolio. The authors observed how the risk structure of a portfolio changes over time based on the developed model. Gurgul and Machno (2016) investigated the interdependence of financial markets among twelve European countries and among twelve Asia-Pacific countries using stock market indices. The authors utilized regime-switching copula. The authors concluded that the model efficiently reflect the asymmetric and heavy tail dependence of financial markets 
among countries that were considered. Pircalabu and Benth (2017) used a regime-switching copula to model the interdependence of electricity market prices among European countries. The authors identified the existence of the tail dependence of the prices.

BenSaida (2018) also analyzed the multi-dimensional dependence of bond markets among US and 11 European countries based on Markov regime-switching vine copula. The author confirmed contagion effect in multiple markets. Otani and Imai (2018) utilized a regime-switching copula to model asymmetric dependence in the stock and bond markets of two countries. They discussed characteristics of skew t-copula with vine copula for modeling dependent structures. Rui (2019) applied regime-switching copula to model the dependence of mortality rates using regular vine copula. The author applied the developed model to analyze risk in longevity bonds. Shahzad et al. (2019) analyzed the dependence between the Islamic bond index and benchmark portfolio of Islamic stocks using historical daily data with time-varying regime-switching copula

This study applied a regime-switching copula to analyze a step-down ELS. The variations of the two underlying stock indices- the Eurostoxx50 and the HSCEI of the step-down ELS were modeled with regime-switching copula using well-known copula functions. It is expected that a regimeswitching copula will be added to the list of suitable models used for risk analysis of step-down ELS given that the model has great flexibility in accommodating the complicated dependent structure of two indices.

The remainder of this paper is organized as follows. Section 2 illustrates a typical structure of step-down ELS. Section 3 discusses the framework of the regime-switching copula model. Section 4 briefly introduces the empirical data used for model construction. Section 5 describes the step-wise model selection procedure. Section 6 illustrates the risk analysis for the step-down ELS based on the constructed model. The paper closes with concluding remarks in Section 7.

\section{Structure of step-down ELS}

The step-down ELS is a structured product that has an automatic call feature on prescribed dates called auto-call dates. On each auto-call date, there are two possibilities depending on if the underlying index values (there are usually two or more underlying indices) satisfy the condition for auto-call. If the values of the underlying indices satisfy the condition for auto-call, the security is called and a payment consisting of principal and accrued interest (known as the coupon) over the life of the contract is made. Otherwise, the contract continues without payment. The rate of coupon payment, which applies to the amount initially invested, is prescribed at the beginning of the contract.

A typical step-down ELS that has been popular in Korean financial markets is described and discussed below. It is a three-year maturity step-down ELS for which the underlying indices are the Eurostoxx50 and the HSCEI. The contract is auto-callable at the end of every six months with an annual coupon rate of $6 \%$. At the beginning of the contract, the value of each index is set at 100 and the future value of each index is converted to a percentage of the initial value of the index. Therefore, the converted value of each index represents the level of index value when compared with the initial value of the index. The underlying value of step-down ELS is then defined as the minimum of the two converted values of the indices.

If the underlying value is above the threshold, which decreases over auto-call dates, a redemption payment is made. For example, assume that the thresholds are 90 at the $1^{\text {st }}$ and $2^{\text {nd }}$ auto-call dates, 85 at the next two auto-call dates, and 80 at the final two auto-call dates. Additionally, there is a "KnockIn" barrier (KI) that allows for payment at expiration if no index value, converted as a percentage of initial value of each index, falls below KI over the entire life of the contract, even if the underlying 


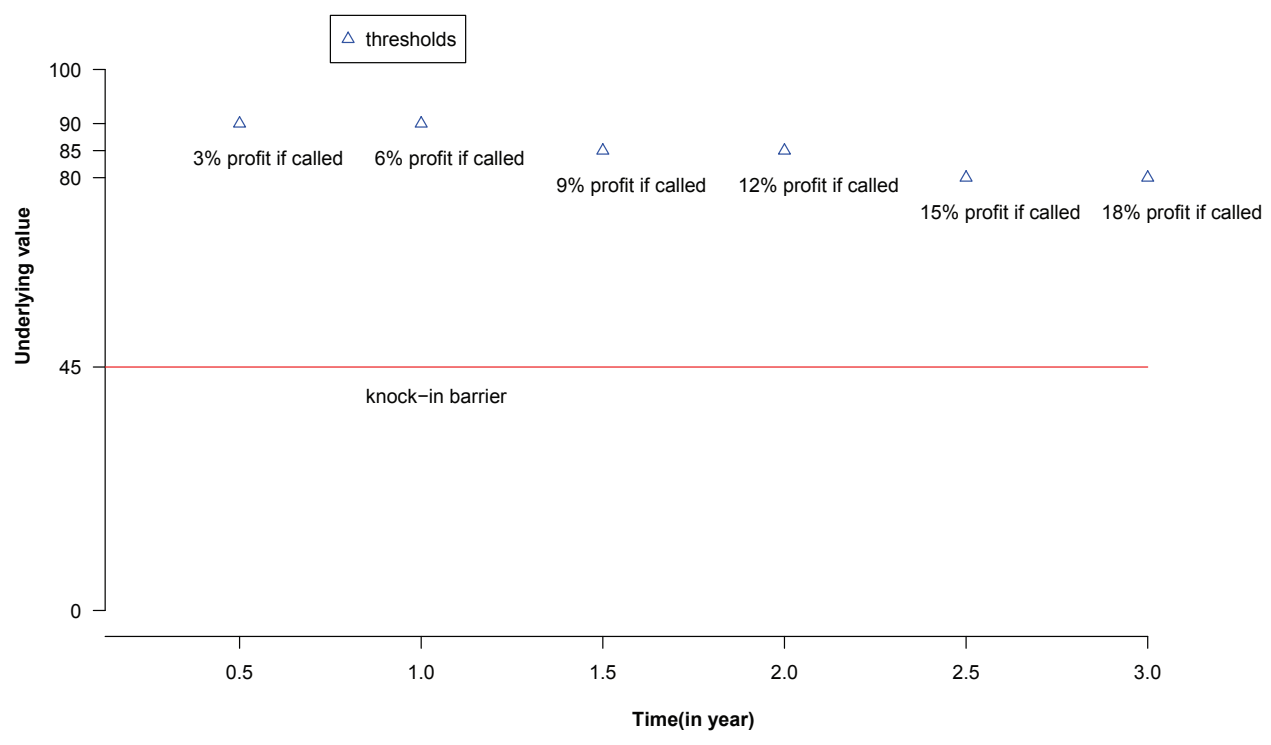

Figure 1: Structure of a step-down equity-linked securities.

value never meets the threshold condition until expiration. For illustration, assume that KI is 45 .

The contract described above is denoted by $90-90-85-85-80-80 / 45 \mathrm{KI}$. Figure 1 illustrates the conditions for auto-call at the end of every six months, together with a knock-in barrier for step-down ELS with 90-90-85-85-80-80/45KI when an initial investment of 1 is made. The payoff structure of the step-down ELS can be summarized as:

- On the first auto-call date, if the underlying value is above 90 , the principal is redeemed with the accrued coupon for six months, which is $3 \%$ of the initial investment, and the contract is closed. Otherwise, the contract proceeds to the next auto-call date.

- If $k^{\text {th }}$ auto-call date is the first time point at which the underlying value is above the given threshold, then the principal is redeemed with the accrued coupon ( $3 \mathrm{k} \%$ of the initial investment) and the contract is closed.

- If conditions for auto-call are never met prior to expiration, one more chance exists based on the knock-in barrier. If the lowest value of the underlying value over the three years does not fall below 45 , the principal is redeemed with the accrued coupon (18\% of the initial investment) at expiration.

- If conditions for auto-call are never met until the expiration and the lowest value of the underlying value fell below 45 at some point over three years, the percentage indicated by the underlying value of initial investment is redeemed at expiration. That is, investors are faced with a loss ranging from $-20 \%$ to $-100 \%$.

As described, the timing and amount of future payments of a step-down ELS depend on the random variation of underlying indices. Therefore, the construction of a model that is able to capture the characteristics of the movements in underlying indices' values is the cornerstone for risk analysis of step-down ELS. 


\section{Regime-switching copula}

\subsection{Copula}

Consider two random variables $X_{1}$ and $X_{2}$ that have joint distribution $H\left(x_{1}, x_{2}\right)$ with marginal distribution functions $F_{1}\left(x_{1}\right), F_{2}\left(x_{2}\right)$. A bivariate copula $C\left(u_{1}, u_{2}\right)$ is the function that maps an ordered pair $\left(F_{1}\left(x_{1}\right), F_{2}\left(x_{2}\right)\right)$ to $H\left(x_{1}, x_{2}\right)$ such as

$$
H\left(x_{1}, x_{2}\right)=C\left(F_{1}\left(x_{1}\right), F_{2}\left(x_{2}\right)\right) .
$$

The joint density function of $x_{1}$ and $x_{2}$ is expressed as

$$
h\left(x_{1}, x_{2}\right)=c\left(F_{1}\left(x_{1}\right), F_{2}\left(x_{2}\right)\right) \prod_{i=1}^{2} f_{i}\left(x_{i}\right)
$$

where $c\left(u_{1}, u_{2}\right)$ is copula density, which is $c\left(u_{1}, u_{2}\right)=\partial C\left(u_{1}, u_{2}\right) /\left(\partial u_{1} \partial u_{2}\right)$, and $f_{i}\left(x_{i}\right)$ is probability density function corresponding to $F_{i}\left(x_{i}\right)$. The explicit functional form of $C\left(u_{1}, u_{2}\right)$ characterizes the dependence structure of two random variables under consideration. In this study, the four following prominent bivariate copula models were considered.

- Gaussian (Normal) copula

$$
C_{N}\left(u_{1}, u_{2} ; \rho\right)=\int_{-\infty}^{\Phi^{-1}\left(u_{1}\right)} \int_{-\infty}^{\Phi^{-1}\left(u_{2}\right)} \frac{1}{2 \pi \sqrt{1-\rho^{2}}} \exp \left\{\frac{-\left(r^{2}-2 \rho r s+s^{2}\right)}{2 \sqrt{1-\rho^{2}}}\right\} d r d s, \quad(-1<\rho<1),
$$

where $\Phi(x)$ is the distribution function of standard normal distribution.

- Student's t-copula

$$
C_{T}\left(u_{1}, u_{2} ; \rho, v\right)=\int_{-\infty}^{t_{\nu}^{-1}\left(u_{1}\right)} \int_{-\infty}^{t_{\nu}^{-1}\left(u_{2}\right)} \frac{1}{2 \pi \sqrt{1-\rho^{2}}}\left(1+\frac{r^{2}-2 \rho r s+s^{2}}{v \sqrt{1-\rho^{2}}}\right)^{-\frac{v+2}{2}} d r d s, \quad(-1<\rho<1, v>0),
$$

where $t_{v}(x)$ is the distribution function of Student's $t$-distribution with degrees of freedom $v$.

- Gumbel copula

$$
C_{G}\left(u_{1}, u_{2} ; \theta\right)=\exp \left[-\left\{\left(-\ln u_{1}\right)^{\theta}+\left(-\ln u_{2}\right)^{\theta}\right\}^{\frac{1}{\theta}}\right], \quad(\theta \geq 1) .
$$

- Clayton copula

$$
C_{C}\left(u_{1}, u_{2} ; \theta\right)=\left(u_{1}^{-\theta}+u_{2}^{-\theta}-1\right)^{-\frac{1}{\theta}}, \quad(\theta \geq-1) .
$$

\subsection{Regime-switching log-normal (RSLN) model}

Variations of a stock (index) in one year time interval $(t-1, t)$ are represented by return $R_{t}=X_{t} / X_{t-1}$ $(t=1,2, \ldots)$, where $X_{t}$ is the value of stock (index) at time $t$. Typical approaches for modeling the value of a stock (index) over time are to fit parametric statistical distributions, time series models, and stochastic models using empirical data of stock (index) returns over a number of periods. The log-normal distribution is a popular way to fit the data of returns. 
An expansion of the log-normal distribution is the regime-switching log-normal (RSLN) model that allows for different values of model parameters in different economic conditions. For example, RSLN with two regimes (RSLN-2) can accommodate two different economic conditions in which the uncertainty of a stock return is high (high-volatility regime) or low (low-volatility regime). Perimeters $\mu$ and $\sigma$ of the log-normal distribution are estimated separately under the two regimes. The likelihood of change in regime should then be specified.

Consider the RSLN-2 model where two regimes are indicated by State 1 and State 2 . The parameters of $\log$-normal distribution under State $i(=1,2)$ are denoted by $\mu_{i}$ and $\sigma_{i}$. For discrete time points denoted by $t(=0,1,2, \ldots)$, the change in regime can be specified by a homogeneous discrete-time Markov chain with the following transition matrix

$$
P=\left(\begin{array}{ll}
p_{11} & p_{12} \\
p_{21} & p_{22}
\end{array}\right)
$$

where $p_{i j}$ is the conditional probability that the regime is $j$ after one unit of time period given that the current regime is $i$. For example, if $p_{11}=p_{12}=p_{21}=p_{22}=1 / 2$, maintaining the current regime and moving to the other regime are equally likely at any point of time. Then, for the return $R_{t}$ under the state of regime for the interval, denoted by $s_{t}$, we have

$$
\ln R_{t} \mid s_{t}=j \sim N\left(\mu_{j}, \sigma_{j}\right), \quad j=1,2 .
$$

Therefore, the RSLN-2 model requires the estimation of six parameters. For a more detailed discussion of the RSLN model, refer to Hardy (2003).

\subsection{Regime-switching copula}

As discussed in Section 3.1, copula can be utilized to construct the joint distribution by joining several random variables' marginal distributions in order to reflect their dependence structure. The dependence structure may change according to economic conditions; therefore, the framework of the regime-switching model allows for consideration of various modes of dependence structure in a model. The type of copula, parameters of copula, or both are separately modeled under the several defined regimes.

Consider a regime-switching copula of two random variables, representing two stock indices in two different regions, with two regimes, in which the marginal distributions of the two random variables are given. The degree of dependence and/or dependent structure changes according to the economic environment, which is represented by the two regimes. If the two random variables are the returns of two different stocks on the interval $(t-1, t)$, denoted by $R_{1 t}$ and $R_{2 t}$, whose marginal distribution functions under the state of regime $j$ are $F_{1}^{(j)}(\cdot)$ and $F_{2}^{(j)}(\cdot)$, and corresponding density functions are $f_{1}^{(j)}(\cdot)$ and $f_{2}^{(j)}(\cdot)$, respectively, then the joint distribution of $R_{1 t}$ and $R_{2 t}$ under state of regime $s_{t}(=1,2)$ is expressed as

$$
h_{R_{1 t}, R_{2 t}}\left(r_{1 t}, r_{2 t} \mid s_{t}=j\right)=c^{(j)}\left(F_{1}^{(j)}\left(r_{1 t}\right), F_{2}^{(j)}\left(r_{2 t}\right)\right) \prod_{i=1}^{2} f_{i}^{(j)}\left(r_{i t}\right), \quad j=1,2,
$$

where $c^{(j)}$ is the copula density defined under the state of regime $j=1,2$. Figure 2 illustrates the structure of a regime-switching copula. 


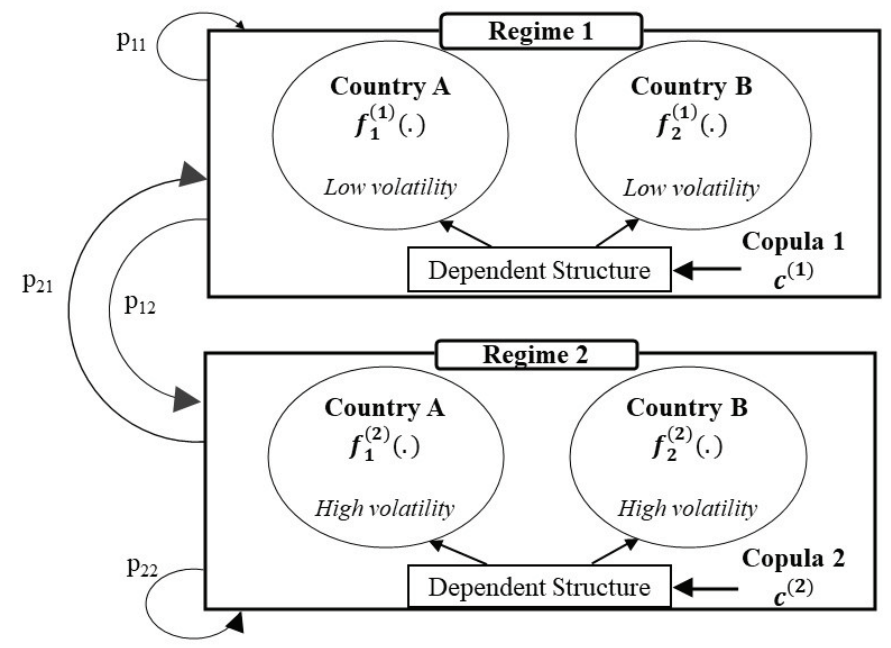

Figure 2: Structure of a regime-switching copula.

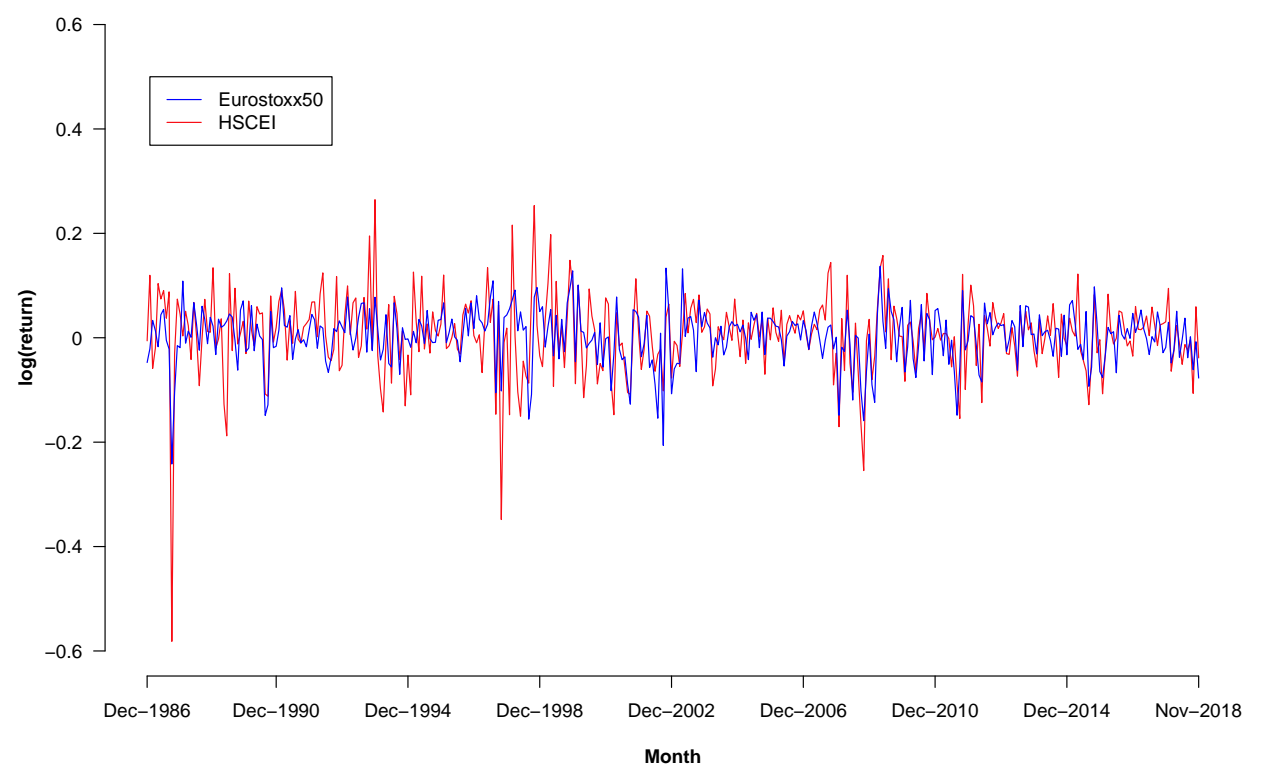

Figure 3: Monthly log-return of Eurostoxx50 and HSCEI (From December 1986 to December 2018).

\section{Data}

The data used for the construction of the regime-switching copula were Eurostoxx 50 and HSCEI monthly index values from November 1986 to November 2018, totaling 385 observations. Based on the data, 384 consecutive values of monthly returns were available for analysis. Figure 3 shows the historical monthly log-returns of the two indices, and Figure 4 presents the frequency of monthly logreturn discretized by interval having length of 0.02 . Table 1 also summarizes descriptive statistics for 

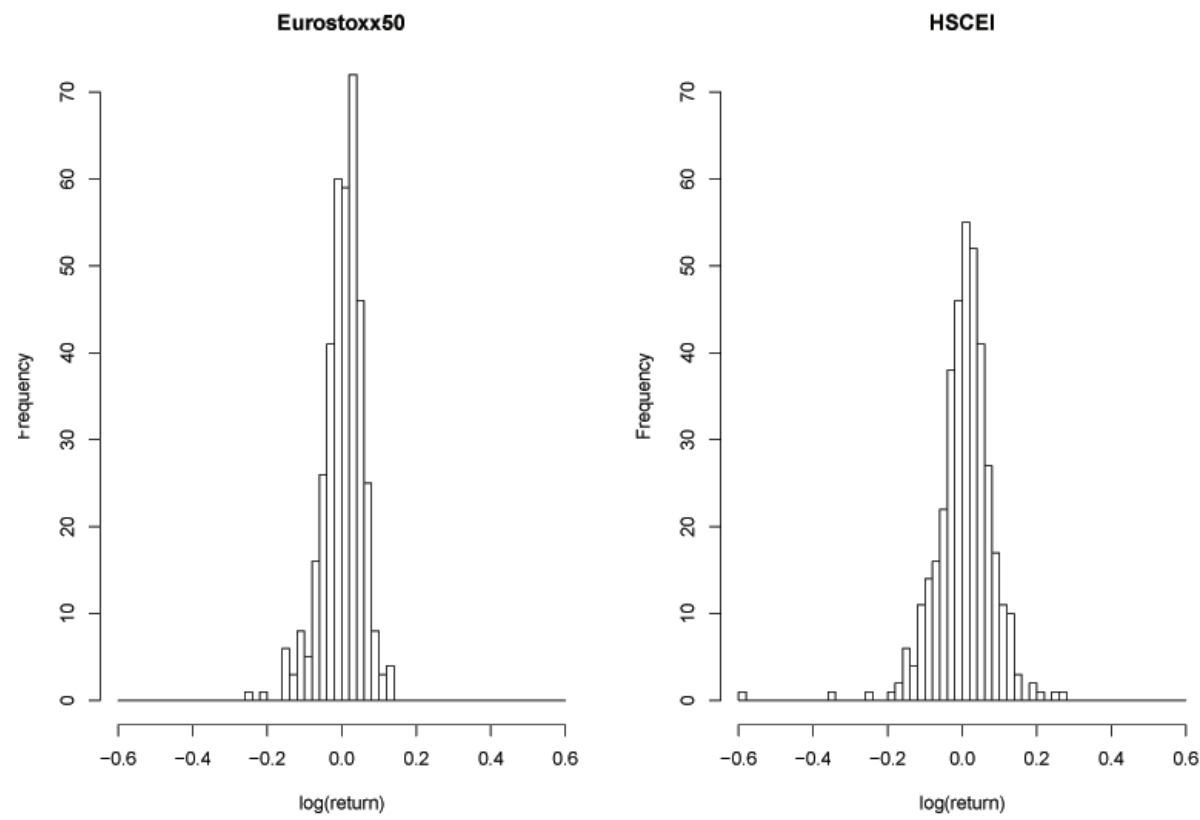

Figure 4: Frequency of log-return of Eurostoxx 50 and HSCEI.

Table 1: Descriptive statistics of monthly log-return of Eurostoxx50 and HSCEI

\begin{tabular}{lrr}
\hline \hline & Eurostoxx50 & HSCEI \\
\hline Mean & 0.0031 & 0.0060 \\
Median & 0.0107 & 0.0104 \\
Maximum & 0.1370 & 0.2645 \\
Minimum & -0.2419 & -0.5816 \\
Standard deviation & 0.0530 & 0.0765 \\
Skewness & -0.8350 & -1.3979 \\
Kurtosis & 4.9446 & 13.0176 \\
\hline \hline
\end{tabular}

the data.

In Figure 3, it is observed that the two stock indices tend to move in the same direction, especially at some points of time when fluctuation is large. The volatility of HSCEI is also larger than the volatility of Eurostoxx 50, which is indicated by maximum, minimum and standard deviation of logreturn in Table 1. The average return of HSCEI is almost twice as large as the average return of Eurostoxx50, which reflects the higher volatility of HSCEI.

Importantly, both series of monthly returns indicated that the data did not seem to fit with a single normal distribution. The frequency histograms in Figure 4 do not look asymmetric and the descriptive statistics such as skewness and kurtosis (Table 1) do not support that the data follow a single normal distribution. It was also found that the variability of a log-return is larger for the HSCEI (Figure 3 and Figure 4). The correlation of log-returns between the two indices was shown in Figure 5. As expected, the corresponding values of log-return show a positive correlation. This supports the existence of dependence between the two indices.

Based on the results from the exploratory data analysis, two important aspects, which should be carefully considered in modeling returns of the two stock indices, were identified. First, the returns of 


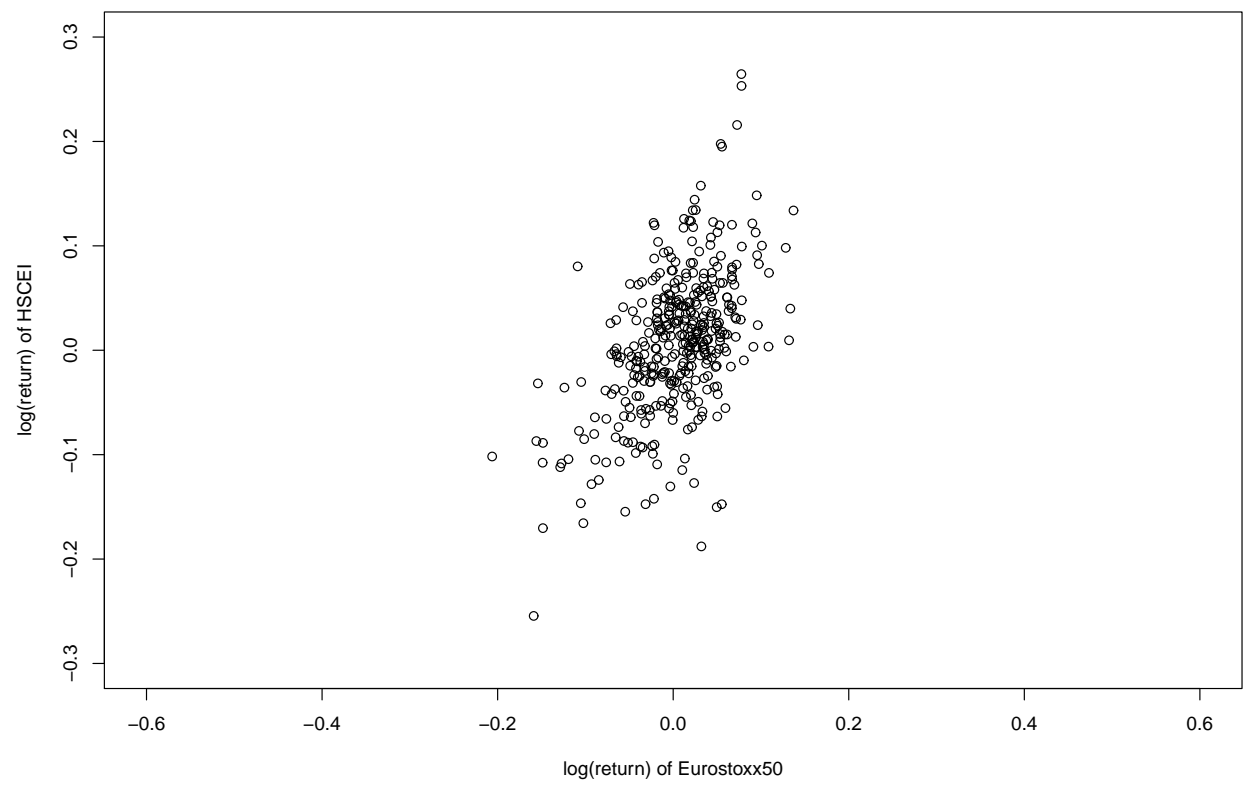

Figure 5: Relationship of monthly log-return between Eurostoxx50 and HSCEI.

the two stock indices have some correlation and the degree of correlation does not always maintain a certain level. Therefore, a desired model should allow me possible change in the degree of dependence. The other is the difference in the characteristics of distribution of log-return for the two stock indices. A model should capture the feature of the movement for each stock index.

The regime-switching copula model described in the previous section is a good candidate for reflecting dependence structure of the two stock indices where the degree of dependence may change over time. The two different types of copula in the regime-switching model are expected to capture tail dependence between the two stock indices more efficiently since the degree of dependence is expected to be larger when returns are a lot higher or lower than average.

\section{Model selection}

As discussed in Section 3, model construction using copula consists of two stages. This is called the Inference Function for Margins (IFM) method. The first stage is to determine marginal models for the returns of the Eurostoxx 50 and HSCEI. Based on the selected models for both indices, a regimeswitching copula model, which is most suitable for reflecting the dependence structure between the two indices, is constructed in the second stage. The procedure for model construction based on data introduced in the previous section as follows.

\subsection{Marginal model}

In order to construct a marginal model for the return of each index, various models that are known to suitably fit stock (index) returns were considered. Among the models, the RSLN-2 model and time series model specified by $\operatorname{ARMA}(p, q)-\operatorname{GARCH}(1,1)$ were found to be candidate models for the data that was to be fitted. Model parameter estimation was then performed using maximum likelihood 
Table 2: Model comparison for marginal model of each index

\begin{tabular}{clcrrr}
\hline \hline Index & \multicolumn{1}{c}{ Model } & Number of parameters & Log-likelihood & AIC & BIC \\
\hline \multirow{5}{*}{ Eurostoxx50 } & 6 & 614.53 & $-1,217.06$ & $-1,193.34$ \\
& ARMA(1,1)-GARCH(1,1) & 7 & 614.82 & $-1,215.64$ & $-1,187.97$ \\
& ARMA(1,2)-GARCH(1,1) & 7 & 614.64 & $-1,215.28$ & $-1,187.61$ \\
& ARMA(2,1)-GARCH(1,1) & 7 & 614.74 & $-1,215.49$ & $-1,187.81$ \\
& AR(3)-GARCH(1,1) & 8 & 614.97 & $-1,213.94$ & $-1,182.31$ \\
& AR(4)-GARCH(1,1) & 6 & 617.34 & $-1,222.69$ & $-1,198.97$ \\
\hline \multirow{5}{*}{ HSCEI } & RSLN-2 & 6 & 493.26 & -974.53 & -950.81 \\
& ARMA(1,1)-GARCH(1,1) & 7 & 493.56 & -973.13 & -945.45 \\
& ARMA(1,2)-GARCH(1,1) & 7 & 493.52 & -973.05 & -945.37 \\
& ARMA(2,1)-GARCH(1,1) & 7 & 493.19 & -972.37 & -944.70 \\
& AR(3)-GARCH(1,1) & 8 & 493.62 & -971.23 & -939.61 \\
& AR(4)-GARCH(1,1) & 6 & 495.66 & -979.33 & -955.61 \\
\hline \hline
\end{tabular}

Table 3: Estimated parameters of RSLN-2 models

\begin{tabular}{ccc}
\hline \hline \multirow{2}{*}{ Parameters } & Index & HSCEI \\
\cline { 2 - 3 } & Eurostoxx 50 & $0.0185(0.2226)$ \\
$\mu_{1}$ & $0.0142(0.1709)$ & $-0.0082(-0.0988)$ \\
$\mu_{2}$ & $-0.0211(-0.2527)$ & $0.0486(0.1685)$ \\
$\sigma_{1}$ & $0.0342(0.1184)$ & $0.1165(0.4036)$ \\
$\sigma_{2}$ & $0.0841(0.2912)$ & 0.0464 \\
$p_{12}$ & 0.0570 & 0.0633 \\
$p_{21}$ & 0.1418 & \\
\hline \hline
\end{tabular}

estimation. Table 2 presents the resulting information from the estimated candidate models for model comparison.

The RSLN-2 model turned out to be the best model based on the Akaike information criterion (AIC) and Bayesian information criterion (BIC). Table 3 shows the estimated parameters of the RSLN-2 model. As the models are based on monthly data, the annualized parameters for $\mu$ and $\sigma$ are provided in parenthesis. The probability of being in the high-volatility regime (State 2) in each month of the data period was calculated to observe how correlated variations of the two indices were reflected in the estimated RSLN-2 model. If $r_{t}$ and $s_{t}$ are the realized return and the state of regime in the $t^{t h}$ month, respectively, the probability of being in State 2 can be estimated by the following recursion.

$$
\begin{gathered}
\operatorname{Pr}\left(s_{1}=i \mid r_{1}\right)=\frac{\operatorname{Pr}\left(r_{1} \mid s_{1}=i\right) \cdot \operatorname{Pr}\left(\pi_{1} p_{1 i}+\pi_{2} p_{2 i}\right)}{\sum_{k=1}^{2} \operatorname{Pr}\left(r_{1} \mid s_{1}=k\right) \cdot \operatorname{Pr}\left(\pi_{1} p_{1 k}+\pi_{2} p_{2 k}\right)}, \quad i=1,2, \\
\operatorname{Pr}\left(s_{t+1}=i \mid r_{t+1}, \ldots, r_{1}\right)=\frac{\operatorname{Pr}\left(r_{t+1} \mid s_{t+1}=i\right) \sum_{k=1}^{2} p_{k i} \cdot \operatorname{Pr}\left(s_{t}=k \mid r_{t}, \ldots, r_{1}\right)}{\sum_{i=1}^{2} \operatorname{Pr}\left(r_{t+1} \mid s_{t+1}=i\right) \sum_{k=1}^{2} p_{k i} \cdot \operatorname{Pr}\left(s_{t}=k \mid r_{t}, \ldots, r_{1}\right)}, \quad i=1,2 .
\end{gathered}
$$

Figure 6 presents the results. We observe that the overall shape of probabilities of being in regime 2 by month is similar for the two indices. It indicates the interdependence of the two indices; however, the degree of dependence is not always the same.

According to the model, the Eurostoxx50 has experienced long periods of the high-volatility regime from 1996 to 2002 and from 2006 to 2016. These periods correspond to the US financial crisis in 2008 and the European Crisis in 2013-2014. Likewise, the HSCEI has been under a highvolatility regime from 1986 to 1998 and from 2006 to 2011, which are attributable to periods of financial turbulence such as the IMF crisis in 1997 and the dotcom bubble explosion. 
Eurostoxx50

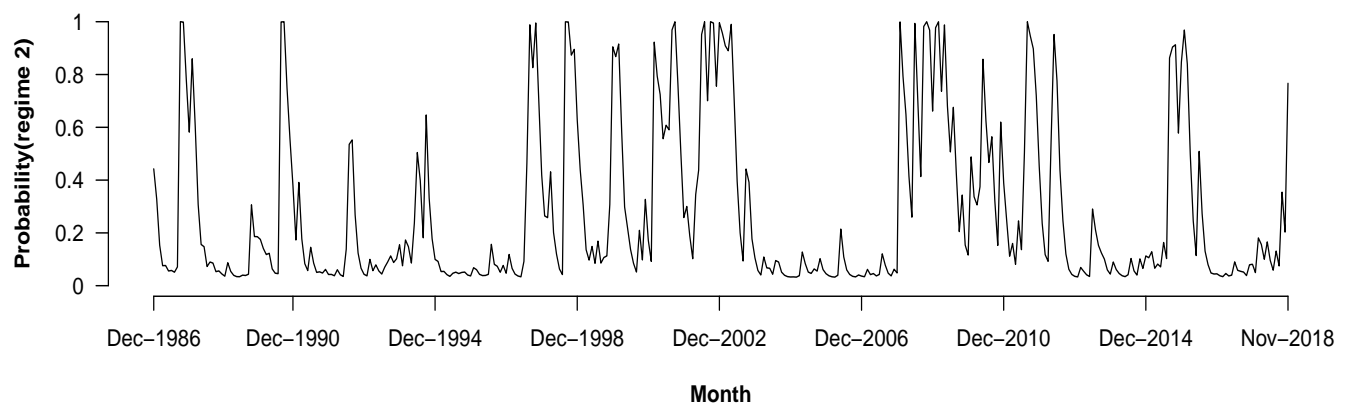

HSCEI

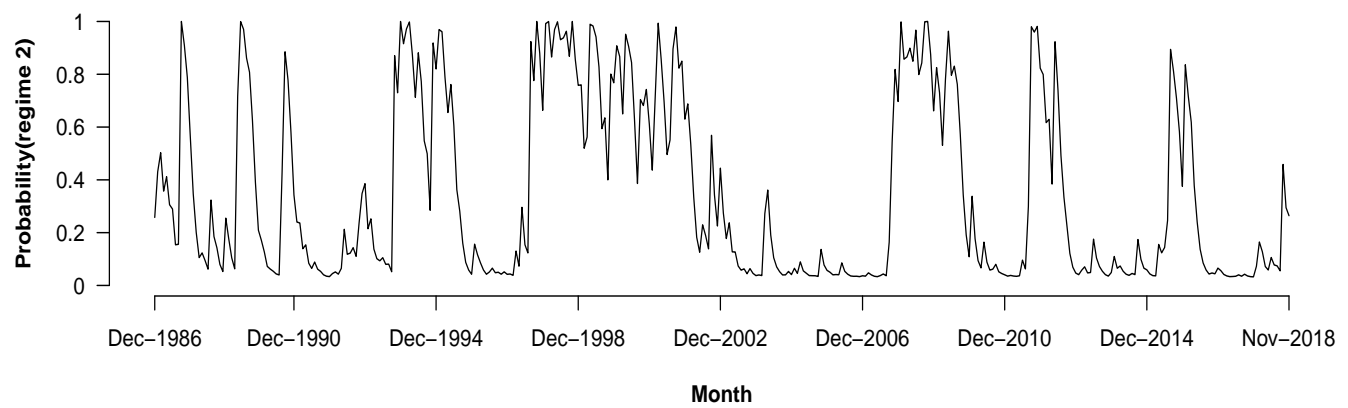

Figure 6: The probability of being in a high-volatility regime derived by RSLN-2 models.

Based on the observations of the behavior of returns for the two stock indices, it was assumed that the two stock indices follow an identical regime change corresponding to the volatility level, which is mainly affected by international economic conditions. The dependence structure of returns of the two indices changes according to the volatility level. We anticipate the model will reflect that the degree of dependence increases as the volatility level increases. For example, an economic event that affects a wider range of regions in the world is more likely to increase the volatility of financial markets in each country and the degree of dependence.

\subsection{Regime-switching copula}

Based on the developed marginal models for the two indices, a regime-switching copula model was calibrated to the entirety of the data. As discussed in Section 3.1, four types of copula (Gaussian, Student's t, Clayton, and Gumbel) were considered for each regime. Given that two regimes were under consideration for which the state of regime at time $t$ is denoted by $s_{t}(=1,2)$, a total of sixteen combinations for the two regimes were modeled and compared. $r_{1 t}$ and $r_{2 t}$ are the realized return of the Eurostoxx50 and HSCEI, respectively, in the $t^{t h}$ month $(t=1,2, \ldots, 364)$ of the data period. $F_{1}^{(j)}\left(r_{1}\right)$ and $F_{2}^{(j)}\left(r_{2}\right)$ denote the distribution functions, specified by RSLN-2, of returns for the Eurostoxx 50 and HSCEI, respectively under regime $j$, while $f_{1}^{(j)}\left(r_{1}\right)$ and $f_{2}^{(j)}\left(r_{2}\right)$ denote the corresponding probability density functions under regime $j$. We then consider the regime-switching copula which is expressed 


$$
h\left(r_{1 t}, r_{2 t} \mid s_{t}=j\right)=c^{(j)}\left(F_{1}^{(j)}\left(r_{1 t}\right), F_{2}^{(j)}\left(r_{2 t}\right)\right) \prod_{i=1}^{2} f_{i}^{(j)}\left(r_{i t}\right), \quad j=1,2,
$$

where $c^{(j)}\left(u_{1}, u_{2}\right)$ is the copula density defined under the state of regime $j(=1,2)$. Consequently, the likelihood contribution of returns in the $t^{\text {th }}$ month, $\left(r_{1 t}, r_{12}\right)$ is expressed as

$$
c\left(F_{1}\left(r_{1 t}\right), F_{2}\left(r_{2 t}\right)\right) \prod_{i=1}^{2} f_{i}\left(r_{i t}\right)
$$

where

$$
\begin{aligned}
c\left(F_{1}\left(r_{1 t}\right), F_{2}\left(r_{2 t}\right)\right)= & c^{(1)}\left(F_{1}^{(1)}\left(r_{1 t}\right), F_{2}^{(1)}\left(r_{2 t}\right)\right) \cdot \operatorname{Pr}\left(s_{t}=1 \mid s_{t-1}, r_{1,(t-1)}, r_{2,(t-1)}, \ldots, r_{1,1}, r_{2,1}\right) \\
& +c^{(2)}\left(F_{1}^{(2)}\left(r_{1 t}\right), F_{2}^{(2)}\left(r_{2 t}\right)\right) \cdot \operatorname{Pr}\left(s_{t}=2 \mid s_{t-1}, r_{1,(t-1)}, r_{2,(t-1)}, \ldots, r_{1,1}, r_{2,1}\right), \\
f_{i}\left(r_{i t}\right)= & f_{i}^{(1)}\left(r_{i t}\right) \cdot \operatorname{Pr}\left(s_{t}=1 \mid s_{t-1}, r_{1,(t-1)}, r_{2,(t-1)}, \ldots, r_{1,1}, r_{2,1}\right) \\
& +f_{i}^{(2)}\left(r_{i t}\right) \cdot \operatorname{Pr}\left(s_{t}=2 \mid s_{t-1}, r_{1,(t-1)}, r_{2,(t-1)}, \ldots, r_{1,1}, r_{2,1}\right) .
\end{aligned}
$$

Notice that the copula density depends on the regime. The probability of being in a certain regime in the $t^{\text {th }}$ month based on historical information, $\operatorname{Pr}\left(s_{t}=1 \mid s_{t-1}, r_{1,(t-1)}, r_{2,(t-1)}, \ldots, r_{1,1}, r_{2,1}\right)$ and $\operatorname{Pr}\left(s_{t}=\right.$ $\left.2 \mid s_{t-1}, r_{1,(t-1)}, r_{2,(t-1)}, \ldots, r_{1,1}, r_{2,1}\right)$, are expressed recursively starting with the stationary probabilities $\operatorname{Pr}\left(s_{1}=1\right)=\pi_{1}=p_{21} /\left(p_{12}+p_{21}\right)$ and $\operatorname{Pr}\left(s_{1}=2\right)=\pi_{2}=p_{12} /\left(p_{12}+p_{21}\right)$. Then, the log-likelihood function $l(\underline{\theta})$, where $\underline{\theta}$ is the parameter vector consisting of parameters in copulas corresponding to the two regimes and the transition probability between the two regimes, to be maximized is as follows.

$$
l(\underline{\theta})=\sum_{t=1}^{384} \ln f_{1}\left(r_{1 t}\right)+\sum_{t=1}^{384} \ln f_{2}\left(r_{2 t}\right)+\sum_{t=1}^{384} \ln c\left(F_{1}\left(r_{1 t}\right), F_{2}\left(r_{2 t}\right)\right) .
$$

The first two terms in the equation are the log-likelihood of the RSLN-2 model for the two indices' returns. The estimated parameters in the sixteen models and their AIC and BIC values are presented in Table 4. The best fitting model based on both AIC and BIC was the regime-switching copula with a Gaussian copula for low-volatility regime and Student's t-copula for a high-volatility regime. The dependence measure of two random variables modeled by copula is represented by Kendall's tau. Kendall's tau of the Gaussian and Student's t-copula is $2 / \pi \arcsin (\rho)$. The calculated value of Kendall's tau is 0.14075 for regime 1 and 0.45424 for regime 2. The parameters indicating correlation in the selected model also show that a higher correlation is associated with regime $2(\rho=0.2193$ in regime 1 and $\rho=0.6545$ in regime 2). Therefore, the suggested model desirably reflects that the degree of dependence is larger in periods of high-volatility as observed in Section 4.

\section{Risk analysis}

Based on the selected model in the previous section, the step-down ELS were analyzed. As discussed in Section 2, three-year step-down ELS that are auto-callable every six months, with a 90-90-85-8580-80/45KI structure and based on the minimum of the Eurostoxx50 and HSCEI, were considered. Index values of the Eurostoxx50 and HSCEI at time $t$ (in years) are denoted by $S_{t}^{\text {EURO }}$ and $S_{t}^{\mathrm{HSCEI}}$, 
Table 4: Comparison of regime-switching copula models

\begin{tabular}{|c|c|c|c|c|c|c|}
\hline \multirow{2}{*}{$\begin{array}{c}\text { Models } \\
\text { (Regimes 1/2) }\end{array}$} & \multicolumn{4}{|c|}{ Parameters } & \multicolumn{2}{|c|}{ Model selection criteria } \\
\hline & Regime 1 & Regime 2 & $p_{11}$ & $p_{22}$ & AIC & $\mathrm{BIC}$ \\
\hline $\mathrm{G} / \mathrm{G}$ & $\rho=0.6569$ & $\rho=0.2185$ & 0.9378 & 0.9016 & -90.3748 & -70.6216 \\
\hline $\mathrm{G} / \mathrm{T}$ & $\rho=0.2193$ & $\rho=0.6545, \nu=89.8581$ & 0.9383 & 0.9017 & -98.3154 & -84.5396 \\
\hline $\mathrm{G} / \mathrm{Gu}$ & $\rho=0.4290$ & $\theta=2.3635$ & 0.9794 & 0.8527 & -83.5946 & -63.8413 \\
\hline $\mathrm{G} / \mathrm{C}$ & $\rho=0.6584$ & $\theta=0.2993$ & 0.9362 & 0.9051 & -90.1818 & -70.4286 \\
\hline $\mathrm{T} / \mathrm{T}$ & $\rho=0.6540, v=99.4768$ & $\rho=0.2204, v=99.615$ & 0.9390 & 0.9034 & -86.2521 & -58.5976 \\
\hline $\mathrm{T} / \mathrm{G}$ & $\rho=0.6543, v=89.8582$ & $\rho=0.2194$ & 0.9016 & 0.9383 & -88.1154 & -64.6115 \\
\hline $\mathrm{T} / \mathrm{Gu}$ & $\rho=0.6249, v=92.4179$ & $\theta=1.0604$ & 0.9507 & 0.8722 & -86.9155 & -63.2116 \\
\hline $\mathrm{T} / \mathrm{C}$ & $\rho=0.6558, v=93.9441$ & $\theta=0.2989$ & 0.9379 & 0.9063 & -88.1067 & -64.4037 \\
\hline $\mathrm{Gu} / \mathrm{Gu}$ & $\theta=1.7506$ & $\theta=1.1356$ & 0.9416 & 0.9225 & -73.2385 & -53.4852 \\
\hline $\mathrm{Gu} / \mathrm{G}$ & $\theta=2.3635$ & $\rho=0.4290$ & 0.8527 & 0.9794 & -83.5946 & -63.8414 \\
\hline $\mathrm{Gu} / \mathrm{T}$ & $\theta=1.0604$ & $\rho=0.6249, v=92.4183$ & 0.8722 & 0.9507 & -86.9155 & -63.2116 \\
\hline $\mathrm{Gu} / \mathrm{C}$ & $\theta=1.3230$ & $\theta=1.0117$ & 0.8733 & 0.8856 & -83.8168 & -64.0636 \\
\hline $\mathrm{C} / \mathrm{C}$ & $\theta=0.9536$ & $\theta=0.1171$ & 0.9611 & 0.8488 & -76.4824 & -56.7292 \\
\hline $\mathrm{C} / \mathrm{G}$ & $\theta=0.5753$ & $\rho=0.4897$ & 0.9701 & 0.9981 & -87.1585 & -67.4053 \\
\hline $\mathrm{C} / \mathrm{T}$ & $\theta=0.5230$ & $\rho=0.4901, v=86.368$ & 0.9754 & 0.9503 & -86.3036 & -62.6025 \\
\hline $\mathrm{C} / \mathrm{Gu}$ & $\theta=1.0287$ & $\theta=1.3171$ & 0.8787 & 0.8660 & -83.7775 & -64.0242 \\
\hline
\end{tabular}

$\mathrm{G}=$ Gaussian copula, $\mathrm{T}=$ Student's t-copula, $\mathrm{Gu}=$ Gumbel copula, $\mathrm{C}=$ Clayton copula.

$\mathrm{AIC}=$ Akaike information criterion; $\mathrm{BIC}=$ Bayesian information criterion.

Table 5: Estimated probabilities that redemption payment is made under regime-switching copula

\begin{tabular}{cccc}
\hline \hline Time of Payment & Redemption Payment & Condition & Probability \\
\hline 0.5 & 103 & auto-call (90) & 0.7703 \\
1.0 & 106 & auto-call (90) & 0.1048 \\
1.5 & 109 & auto-call (85) & 0.0409 \\
2.0 & 112 & auto-call (85) & 0.0164 \\
2.5 & 115 & auto-call (80) & 0.0182 \\
3.0 & 118 & auto-call (80) & 0.0095 \\
3.0 & 118 & knock-in (45) & 0.0080 \\
\hline
\end{tabular}

respectively, and the threshold at time $t$ is denoted by $K_{t}$. Letting $S_{t}=\min \left(S_{t}^{\mathrm{EURO}}, S_{t}^{\mathrm{HSCEI}}\right)$. Additionally, $S_{0}^{\text {EURO }}=S_{0}^{\mathrm{HSCEI}}=100$ and if the contract is still in-force at time $t(=0.5,1,1.5,2,2.5,3)$ and $S_{t}>K_{t}$, the payoff when it is auto-called is $100(1+0.06 \times t)$. If at contract expiration at the end of three years $S_{t}$ has never been below the knock-in barrier of 45 over the life of the contract, the payoff is 118 . Otherwise, the investor will receive $S_{3}$ at expiration resulting in investment loss.

The risk for the step-down ELS provider is based on the possibility that payment is made at each time point since the accrued coupon payments are fixed at each auto-call date and at expiration. In order to estimate the probability that the coupon payment is made at the end of every six months, the variations in values of $S_{t}^{\mathrm{EURO}}$ and $S_{t}^{\mathrm{HSCEI}}$ over the life of the contract were simulated using the developed regime-switching copula in the previous section.

Based on the regime-switching copula, monthly returns of each index can be generated. As a result, sample paths for each index over the life of the contract can be derived; consequently, 10,000 sample paths were generated for simulation. When, the sample paths were generated, the starting regime was generated based on $\pi_{1}$ and $\pi_{2}$. Table 5 summarizes the estimated probability of redemption due to auto-call and the probability of redemption at expiration together with probability of investment loss.

Out of 10,000 simulation results, 319 cases ended up with an investment loss as the minimum of the two stock indices never satisfied the payment conditions presented in Table 5. As exhibited, the probability that a redemption payment is made shows a decreasing pattern over time. Therefore, an 


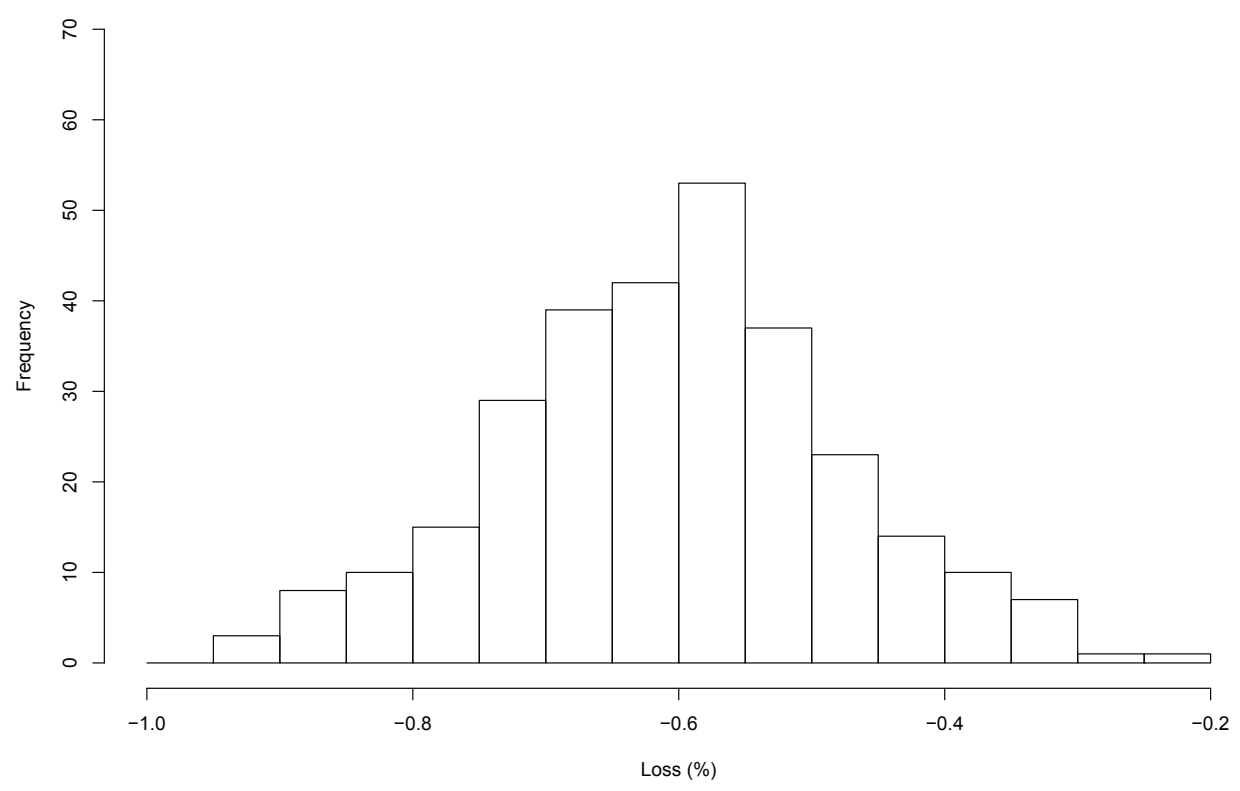

Figure 7: Distribution of loss.

investor should expect that they are likely to receive a redemption payment before expiration and the step-down ELS issuer should expect the cash flow for a redemption payment to be concentrated at the early stage of the contract.

Table 5 shows that there is a $91.6 \%$ chance that a redemption payment is made following 1.5 years of the contract. It is also estimated that the probability of redemption before expiration is $95.1 \%$. Figure 7 shows the distribution of loss, expressed as a range from $-20 \%$ to $-100 \%$ for simulation scenarios that resulted in a loss. The mean average loss in these cases is $-60.29 \%$. The loss distribution presented in Figure 7 shows that there is also a greater probability in a larger loss. Therefore, the size of loss is expected to be fairly significant in the case of loss.

It is important to compare how the estimated probabilities that redemption is made at each autocall date (including the case where the knock-in feature of the contract takes effect) vary across several models. The models used for comparison are lognormal (LN) distribution and the RSLN-2 model. The models were fitted by maximum likelihood estimation. Table 6 presents the probabilities that a redemption payment is made at each auto-call date and that payment is made with the knock-in feature together with the probability of loss.

The model with lognormal distribution considers only one regime and the independent behavior of the two indices. In this model, the minimum value of the two stock indices is expected to be attained by one specific stock index. If regime-switching model for each stock index is considered, dependence between the two stock indices can be introduced as observed in Figure 6. That is, high volatility regime is associated with a global or economic situation that affects most countries in the world simultaneously. Finally, a stronger degree of dependence can be considered when a regimeswitching copula is used. The degree of dependence is stronger in the period of high dependence regime (regime 2 in the model) since the volatility regime of marginal models in the copula is directly associated with the dependence regime (copula regime).

The first observation is that the probability of a redemption payment made at each available time 
Table 6: Model comparison for probability of redemption payment

\begin{tabular}{ccccc}
\hline \hline $\begin{array}{c}\text { Time of } \\
\text { payment }\end{array}$ & Condition & $\begin{array}{c}\text { Independent } \\
\text { lognormal models }\end{array}$ & $\begin{array}{c}\text { Independent } \\
\text { RSLN-2 models }\end{array}$ & $\begin{array}{c}\text { Regime-Switching copula } \\
\text { involving RSLN-2 models }\end{array}$ \\
\hline 0.5 & auto-call (90) & 0.6456 & 0.6576 & 0.7703 \\
1.0 & auto-call (90) & 0.1595 & 0.1590 & 0.1048 \\
1.5 & auto-call (85) & 0.0721 & 0.0631 & 0.0409 \\
2.0 & auto-call (85) & 0.0310 & 0.0306 & 0.0164 \\
2.5 & auto-call (80) & 0.0353 & 0.0295 & 0.0182 \\
3.0 & auto-call (80) & 0.0161 & 0.0114 & 0.0095 \\
3.0 & knock-in (45) & 0.0069 & 0.0075 & 0.0080 \\
\hline \multicolumn{7}{c}{ Investment loss } & 0.0335 & 0.0413 & 0.0319 \\
\hline
\end{tabular}

point varies across the models. The difference of results between the lognormal model and RSLN2 model is relatively small. However, the results obtained from regime-switching copula are quite different from the results derived from the other two models. The main reason that results in the difference is that regime-switching copula considers the dependence between the two indices. This result implies that various models, including models that allow for dependence between underlying indices, should be used to evaluate risk associated with step-down ELS.

The probability that a redemption payment is made based on regime-switching copula tends to be lower than that of the lognormal model and RSLN-2 model, except for a redemption payment at time 0.5 and through the knock-in feature. This result can be justified as the dependence between the underlying two indices are a modeled copula framework. Since the two indices are likely to move in the same direction in regime-switching copula, there is a higher chance that the redemption payment is made at time 0.5 .

Table 5 shows that redemption payments are made at early stage of the contract in lognormal model and RSLN-2 model. The probabilities that the redemption payment is made up to 1.5 years are $87.7 \%, 88.0 \%$, and $91.6 \%$ for lognormal model, RSLN-2 model, and regime-switching copula, respectively. The probabilities that the redemption payment is made before expiration are also $94.4 \%$, $94.0 \%$, and $95.1 \%$ for the lognormal model, RSLN-2 model, and regime-switching copula, respectively. Finally, the probabilities of investment loss are 3.35\%, $4.13 \%$, and 3.19\% for lognormal model, RSLN-2 model, and regime-switching copula, respectively.

Therefore, there are less chances that the minimum value satisfies the condition for redemption payment when two indices move independently. Likewise, the probability that a redemption payment is made according to the knock-in feature is more likely in an independent model since the knockin feature is triggered if either of the two indices reaches the knock-in barrier over the life of the contract. This result implies that the future cash flow corresponding to redemption payments can differ depending on the model. Thus, various candidate models should be compared for risk analysis of step-down ELS to avoid model risk.

The expected present value of payoff can be obtained by simulated returns of the two stock indices using a risk-free rate. However, the present value should not be considered as the price of step-down ELS since the probabilities of redemption were obtained based on experience data, but do not correspond to a risk neutral probability measure.

\section{Concluding results}

A variety of international investment opportunities have evolved due to the globalization of financial markets. Economic interdependence among countries is an important factor that should be considered when making an investment decision associated with international assets. Along these lines, a financial 
product called step-down equity-linked securities (ELS) based on several countries' stock indices, has been of great interest to the Korean investors. Therefore, in the risk analysis of this product, the dependence among the stock indices should be carefully considered.

In this study, a regime-switching copula model was considered in modeling the variations of two stock indices- the Eurostoxx50 and the Hang Seng China Enterprises Index (HSCEI). These indices are commonly used as the underlying assets in the step-down ELS product sold in Korea. The model is able to accommodate the dependence between the two indices as well as the changes in the degree of dependence and/or the dependence structure. Therefore, the regime-switching copula model is a versatile tool to deal with multiple dependent assets.

The regime-switching copula is constructed in two steps. The first step is to select marginal models for the return of each index. The regime-switching lognormal models with two regimes (RSLN-2), representing high and low volatility environments, were selected as marginal models. Using the selected marginal model, the regime-switching copula with two regimes was fitted under the assumption that there are two dependence levels according to the volatility level. After comparing sixteen combinations of four popular copulas, a Gaussian copula for the low volatility regime and a Student's t-copula for the high volatility regime were selected. Our empirical result indicated that low (high) volatility corresponds to low (high) dependence.

A comparison of our model with other popular models showed that the outputs can be quite different across models. In particular, the probabilities that a redemption payment is made at each time point are noticeably different when the models are compared. The result implies that various models should be considered for the risk analysis of step-down ELS. The regime-switching copula is recommended as a good candidate because it is able to reflect the complicated dependence structure of the underlying assets.

Future areas of research include a further exploration for more suitable copula models given that there are a number of available copulas other than the ones discussed in this study. This is expected to improve the risk analysis of step-down ELS. The suggested model in this study is flexible to accommodate the behavior of returns from more than two stock indices as multi-dimensional copula can be implemented when more than two stock indices are used as an underlying asset of step-down ELS. The exploration of a multi-dimensional regime-switching copula model, with various regime structures, can be another area for future research. In addition, analyzing more case studies relating to step-down ELS with different stock indices will provide greater insight on the nature of the dependence structure.

\section{References}

BenSaida A (2018). The contagion effect in European sovereign debt markets: a regime-switching vine copula approach, International Review of Financial Analysis, 58, 153-165.

Cai J (1994). A Markov-model of switching-regime ARCH, Journal of Business and Economic Statistics, 12, 309-316.

Chollete L, Heinen A, and Valdesogo A (2008). Modeling international financial returns with a multivariate regime switching copula, Journal of Financial Econometrics, 7, 437-480.

Delatte AL and Lopez C (2013). Commodity and equity markets: some stylized facts from a copula approach, Journal of Banking and Finance, 37, 5346-5356.

Deng G, Mallett J, and McCann C (2011). Modeling auto-callable structured products, Journal of Derivatives and Hedge Funds, 17, 326-340.

Denuit M, Dhaene J, Goovaerts M, and Kaas R (2005). Actuarial Theory for Dependent Risks: Mea- 
sures, Orders, and Models, Wiley, London.

Genest C and Favre AC (2007). Everything you always wanted to know about copula modelling but were afraid to ask, Journal of Hydrologic Engineering, 12, 347-368.

Guillaume T (2015). Auto-callable structured products, The Journal of Derivatives, 22, 73-94.

Gurgul H and Machno A (2016). Modeling dependence structure among European markets and among Asian-Pacific markets: a regime-switching regular vine copula approach, Central European Journal of Operations Research, 24, 763-786.

Hardy MR (2001). A regime-switching model of long-term stock return, North American Actuarial Journal, 5, 41-53.

Hardy MR (2003). Investment Guarantees: Modeling and Risk Management for Equity-Linked Life Insurance, Wiley, Hoboken.

Jondeau R and Rockinger M (2006). The copula-GARCH model of conditional dependencies: an international stock market application, Journal of International Money and Finance, 25, 827853.

Kang BJ (2016). The investment benefits of structured products: auto-callable equity linked securities, Asian Review of Financial Research, 29, 77-102.

Kim HS and Yeo IK (2011). A case study on the risk of stepdown ELS, The Korean Journal of Applied Statistics, 24, 1021-1031.

Lee H, Ahn S, and Ko B (2019). Generalizing the reflection principle of Brownian motion, and closedform pricing of barrier options and autocallable investments, North American Journal of Economics and Finance, 50, 1-13.

Lee KH, Lim HC, and Choi YS (2016). ELS hedging method based on CVaR using stocks portfolio and futures, Korean Journal of Futures and Options, 24, 423-455.

Lim HC and Choi YS (2015). Knock-in and stocks market effect due to ELS issuance and hedging, Korean Journal of Futures and Options, 23, 289-321.

Nelson RB (2006). An Introduction to Copulas, Springer, New York.

Nguyen C, Bhatti MI, Komornikova M, and Komonik J (2016). Gold price and stock market nexus under mixed-copulas, Economic Modelling, 58, 283-292.

Otani Y and Imai J (2018). An empirical analysis of the dependence structure of international equity and bond markets using regime-switching copula model, International Journal of Applied Mathematics, 48, 191-205.

Pircalabu A and Benth FE (2017). A regime-switching copula approach to modeling day-ahead prices in coupled electricity markets, Energy Economics, 68, 283-302.

Rui Z (2019). Modelling mortality dependence with regime-switching copula, Astin Bulletin, 49, 373407

Schaller H and Norden SV (2010). Regime-switching in stock market returns, Applied Financial Economics, 7, 177-191.

Shahzad SJ, Shahbaz M, Aloui C, and Jammazi R (2019). Are Islamic bonds a good safe haven for stocks? Implications for portfolio management in a time-varying regime-switching copula framework, Applied Economics, 51, 219-238.

Sukcharoen K, Zohrabyan T, Leatham D, and Wu X (2014). Interdependence of oil prices and stock market indices: a copula approach, Energy Economics, 44, 331-339.

Zhu K, Yamaka W, and Sriboonchitta S (2016). Multi-asset portfolio returns: a Markov switching copula-based approach, Thai Journal of Mathematics, 183-200. 
